\title{
Experimental Characterization of Dielectric Properties in Fluid Saturated Artificial Shales
}

\author{
Roman Beloborodov, ${ }^{1}$ Marina Pervukhina, ${ }^{2}$ Tongcheng Han, ${ }^{3,4}$ and Matthew Josh ${ }^{2}$ \\ ${ }^{1}$ Department of Exploration Geophysics, Curtin University, Perth, WA 6151, Australia \\ ${ }^{2}$ CSIRO Energy, Perth, WA 6151, Australia \\ ${ }^{3}$ School of Geosciences, China University of Petroleum (East China), Qingdao 266580, China \\ ${ }^{4}$ Laboratory for Marine Mineral Resources, Qingdao National Laboratory for Marine Science and Technology, Qingdao 266071, China \\ Correspondence should be addressed to Roman Beloborodov; roman.beloborodov@postgrad.curtin.edu.au
}

Received 27 July 2017; Accepted 20 November 2017; Published 20 December 2017

Academic Editor: Feng Yang

Copyright (C 2017 Roman Beloborodov et al. This is an open access article distributed under the Creative Commons Attribution License, which permits unrestricted use, distribution, and reproduction in any medium, provided the original work is properly cited.

\begin{abstract}
High dielectric contrast between water and hydrocarbons provides a useful method for distinguishing between producible layers of reservoir rocks and surrounding media. Dielectric response at high frequencies is related to the moisture content of rocks. Correlations between the dielectric permittivity and specific surface area can be used for the estimation of elastic and geomechanical properties of rocks. Knowledge of dielectric loss-factor and relaxation frequency in shales is critical for the design of techniques for effective hydrocarbon extraction and production from unconventional reservoirs. Although applicability of dielectric measurements is intriguing, the data interpretation is very challenging due to many factors influencing the dielectric response. For instance, dielectric permittivity is determined by mineralogical composition of solid fraction, volumetric content and composition of saturating fluid, rock microstructure and geometrical features of its solid components and pore space, temperature, and pressure. In this experimental study, we investigate the frequency dependent dielectric properties of artificial shale rocks prepared from siltclay mixtures via mechanical compaction. Samples are prepared with various clay contents and pore fluids of different salinity and cation compositions. Measurements of dielectric properties are conducted in two orientations to investigate the dielectric anisotropy as the samples acquire strongly oriented microstructures during the compaction process.
\end{abstract}

\section{Introduction}

Dielectric permittivity of a material is a measure of its frequency dependent electrical polarizability [1], in an applied external electric field. In the presence of an electric field, electrons, ions, and polar molecules all contribute to the frequency dependent dielectric properties. In composite materials, the build-up of charge at dissimilar conductivity boundaries and the creation of exchangeable cations may completely dominate the dielectric behaviour. For example, rock consists of liquid, solid, and gas components and each of them is clearly defined by specific chemical and/or mineral composition. Although the dielectric constants of the most of individual rock components rarely exceed 80 , the bulk dielectric permittivity of a rock sample may reach many orders of magnitude at the radio frequency range (kilohertz to megahertz), implying that not only the constituents of a rock but also their geometry, interaction, spatial arrangement, and interfaces have a significant contribution to polarization [2].

In clay bearing rocks, the presence of a connate brine results in the so-called surface effects: electric double layer polarization (i.e., Stern layer polarization) and MaxwellWagner space-charge polarization. They occur due to the presence of polarizable bound water at the clay-water interface and weakly bounded ions on the surface of mineral particles, which can slowly move under the influence of an external field and contribute toboth the conduction and electric polarization [3]. These effects become prominent at frequencies below $\mathrm{MHz}$ range and complicate the interpretation of data. Figure 1 illustratesthe various polarization effects with the corresponding frequency bands of their occurrence. 


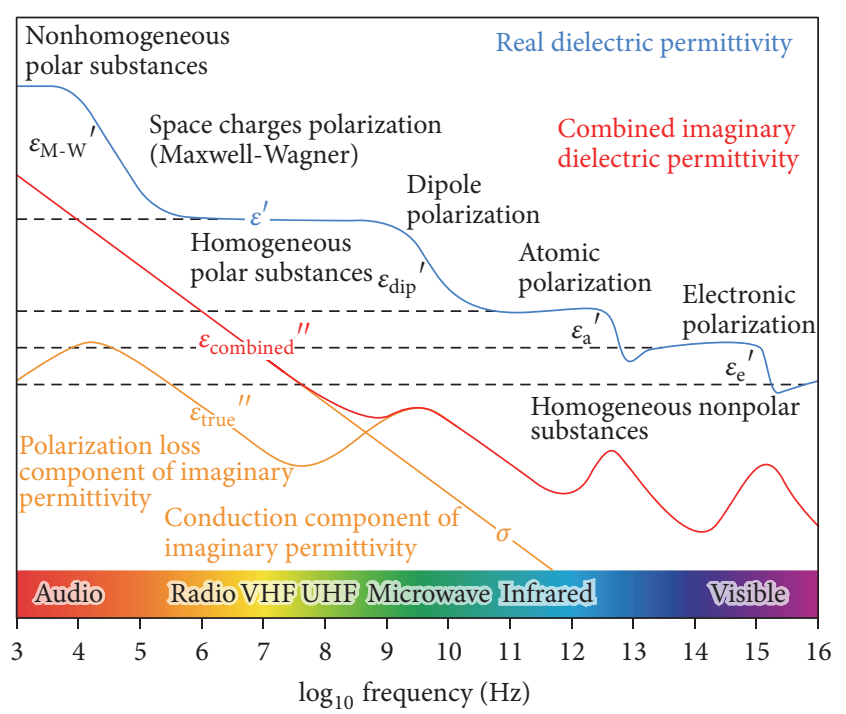

FIGURE 1: Schematics of dielectric polarization/conduction mechanisms, modified from Josh [11].

Dielectric measurements in petrophysics and petroleum industry are mostly used to determine the water content. Water molecules have a fixed electrical dipole moment and rotate quickly to align with an external electric field [4], whereas hydrocarbons have nonpolar molecules and as a result have much lower permittivity than water. Therefore, estimating moisture content from the dielectric permittivity at high frequencies $(>1 \mathrm{GHz})$ is often used in borehole petrophysics. However, dielectric logging at frequencies of $1 \mathrm{GHz}$ and above remains challenging in field measurements due to decreased depth of electric field penetration. On the contrary, currently available commercial dielectric tools operate at multiple spot frequencies [5] to facilitate a determination of water content and CEC: dielectric response at frequencies below $50 \mathrm{MHz}$ is more affected by the surface effects described above [6]. At lower frequencies, clay content, geometry and spatial orientations of mineral rock constituents, and the salinity of pore fluid also affect the measured dielectric permittivity $[7,8]$.

Some recent studies suggest alternative methods for oil recovery in unconventional reservoirs that utilize dielectric heating [9]. This technology allows increasing economical efficiency of exploration and reducing the ecological impact on the environment. It heavily relies on knowledge of the dielectric behaviour in kilohertz-megahertz range of frequencies where the depth of electric field penetration is sufficient for field applications and the dielectric loss-factor exhibits peak values [10]. However, interplay of several physical effects results in wide dispersion of dielectric relaxation peaks at frequencies below gigahertz. Identifying of the frequencies of these attenuation peaks as well as estimation of the range of dielectric dispersion for specific types of rock would be useful for tuning the frequency of dielectric heating tools to ensure the energy efficient and productive heating.

Another purpose of dielectric measurements is the estimation of cation exchange capacity (CEC), a property that is directly attributed to mineral composition and specifically to clay content. Josh [11] showed a strong correlation between dielectric response at $\sim 30 \mathrm{MHz}$ and the CEC. In turn, this property is linearly proportional to the specific surface area (SSA) of a rock that determines character and amount of interparticle contacts (coordination number) under the given mineral composition and porosity of a rock. Therefore, it is expected that dielectric constant should correlate with both static and dynamic elastic properties of a rock.

It is hard to segregate and independently study different factors influencing the dielectric properties of natural shales due to the complex composition of their mineral and fluid components. There are many experimental and theoretical studies dedicated to frequency dependent dielectric properties of rocks with relation to the geometry of their components (e.g., aspect ratios and orientation of mineral grains and pores), water saturation, and organic content (e.g., $[2,12,13])$. However, there is very little evidence of studies taking into account the clay content, microstructure, and the chemical composition of pore fluid (e.g., $[4,7]$ ). The anisotropy of dielectric properties of shales is crucial for the interpretation of dielectric well-logs especially in deviated wells and may affect the efficiency of production via the dielectric heating in unconventional shale reservoirs $[14,15]$. Again, there is little to no evidence on how the anisotropy of dielectric properties of shales depend on the aforementioned parameters. The least known parameter in terms of its effect on dielectric response is the microstructure of the clay fraction in shales. Different geological settings may significantly affect the microstructure of sediment and consequently its properties [16]. For example, Dong and Wang [17] showed that the kaolinite sediments saturated with water solutions of different $\mathrm{pH}$ exhibit significantly different dielectric spectra due to the different mechanisms of microstructure formation.

In this work, we prepare artificial shale samples using the laboratory mechanical compaction of simple water-based mixtures of kaolinite clay and quartz powder. Our goal is to use these models of natural shales to extend our knowledge on and independently investigate the influence of clay content, microstructure, and the chemical composition of pore fluid on their frequency dependent dielectric properties and anisotropy.

\section{Methodology}

2.1. Sample Preparation. Artificial shale samples were prepared from mineral mixtures via mechanical compaction. In order to simplify the modelled rock and reduce uncertainties related to multiphase composition, we chose simplistic mineral components and pore fluid chemicals. Quartz and kaolinite were used for silt and clay fractions as they are the common minerals in natural shales. Compared with minerals from smectite group, kaolinite is nonswelling clay, which makes it one of the easiest clay minerals to work with. Crushed quartz powder consists of silt-sized grains.

To prepare mixtures with different types of initial clay microstructure, we utilized physicochemical approach. The presence of electrolyte in the pore fluid leads to aggregation 
TABLE 1: Specification of the samples.

\begin{tabular}{|c|c|c|c|c|c|}
\hline Name & Type of clay microstructure & Clay content $(\%)$ & Porosity (\%) & Salinity $(\mathrm{g} / \mathrm{l})$ & Salt \\
\hline D0100_0 & Dispersed & 100 & 21 & 0 & - \\
\hline D4555_0 & Dispersed & 55 & 10 & 0 & - \\
\hline $\mathrm{C} 4$ & Dispersed & 75 & 28 & 0 & - \\
\hline C5 & Dispersed & 60 & 23 & 0 & - \\
\hline A1090_0 & Aggregated & 90 & 13 & 0 & - \\
\hline A0100_10 & Aggregated & 100 & 10 & 10 & $\mathrm{NaCl}$ \\
\hline A0100_34 & Aggregated & 100 & 26 & 34 & $\mathrm{NaCl}$ \\
\hline A0100_75 & Aggregated & 100 & 18 & 75 & $\mathrm{KCl}$ \\
\hline A2575_75 & Aggregated & 75 & 16 & 75 & $\mathrm{KCl}$ \\
\hline A4060_75 & Aggregated & 60 & 14 & 75 & $\mathrm{KCl}$ \\
\hline $\mathrm{C} 2$ & Aggregated & 75 & 23 & 75 & $\mathrm{KCl}$ \\
\hline $\mathrm{C} 3$ & Aggregated & 60 & 24 & 75 & $\mathrm{KCl}$ \\
\hline
\end{tabular}

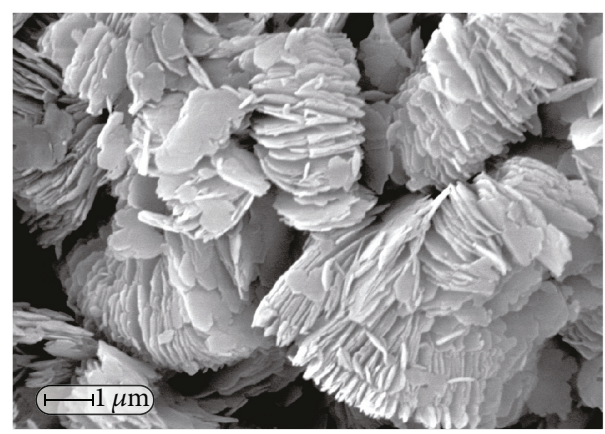

Figure 2: Aggregates of the kaolinite particles.

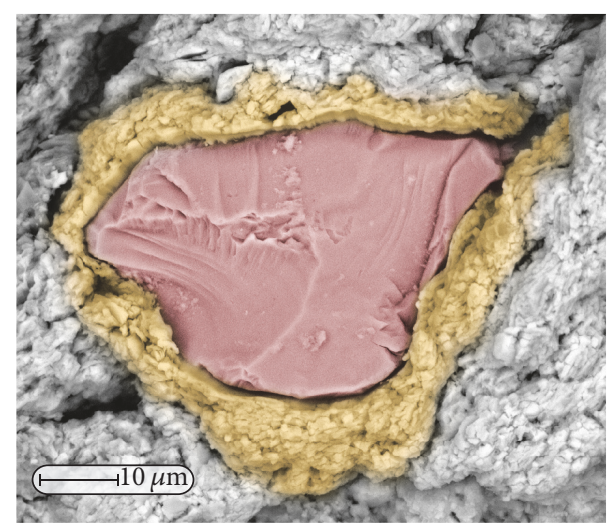

Figure 3: Kaolinite clay coating (in yellow) on the surface of the quartz grain (in red).

of kaolinite clay particles by means of shrinking the diffuse layer of water around the particles and sequential loss of their stability as a colloidal system [19]. Samples with aggregated clay microstructure were prepared by adding a brine solution so that clay platelets and their ultramicroaggregates (basic associations of few axially aligned individual clay platelets) combine together (Figure 2) and form the thick conforming coats on the surfaces of quartz grains (Figure 3 ).
It is important to note that initially untreated kaolinite powder resides in aggregated state. Thus, samples with dispersed clay microstructure were prepared by boiling mixtures with $25 \mathrm{ml}$ of a dispersant - 4\% sodium pyrophosphate tetrabasic, which separates existing clay aggregates into individual clay platelets and their smaller associations. This allows replacing exchangeable cations on the surfaces of clay particles with $\mathrm{Na}^{+}$ion so that its hydrate envelopes repulse and separate clay particles from each other.

Summary of all the prepared mixtures is shown in Table 1. Samples were named using a specific nomenclature where the first letter stands for either aggregated (A) or dispersed (D) clay microstructure; the following numbers indicate the weight ratio of quartz to kaolinite constituents in percentage, and the last number shows the salinity of a pore fluid in $\mathrm{g} / \mathrm{l}$.

\subsection{Mechanical Compaction and Parallel Plate Dielectric Mea-} surements. Samples were compacted in the high-pressure oedometer by applying uniaxial stress. Plastic pistons conduct the stress from the actuator that is manually operated with the hydraulic pump. Cell was designed to safely keep $80 \mathrm{MPa}$ of the vertical stress that corresponds to $\sim 3 \mathrm{~km}$ depth in a sedimentary basin. The oedometer was made of PEEK plastic; this material is strong enough to maintain zero lateral strain. Compacted shales were gently ejected from the oedometer, covered with thick layer of wax, and preserved in a low-temperature humid atmosphere to prevent desiccation. Further details on the mechanical compaction methodology can be found in Beloborodov et al. [16].

A subsample was cut from each of the compacted samples and its dielectric properties are measured in a parallel plate dielectric rig (Figure 4). Electrodes of the dielectric rig are made of brass and they can be changed to match the sample size. For this study, small electrodes of $1 \mathrm{~cm}$ in diameter were used due to the relatively small size of the subsamples $\sim 2 \mathrm{~cm}$ in diameter. To ensure proper coupling between the electrodes of the measurement cell and maintain parallel electric field normal to the faces of the subsample disc, it was polished on a diamond surface grinder to achieve the parallel faces with $30 \mu \mathrm{m}$ tolerance. Thickness of the 


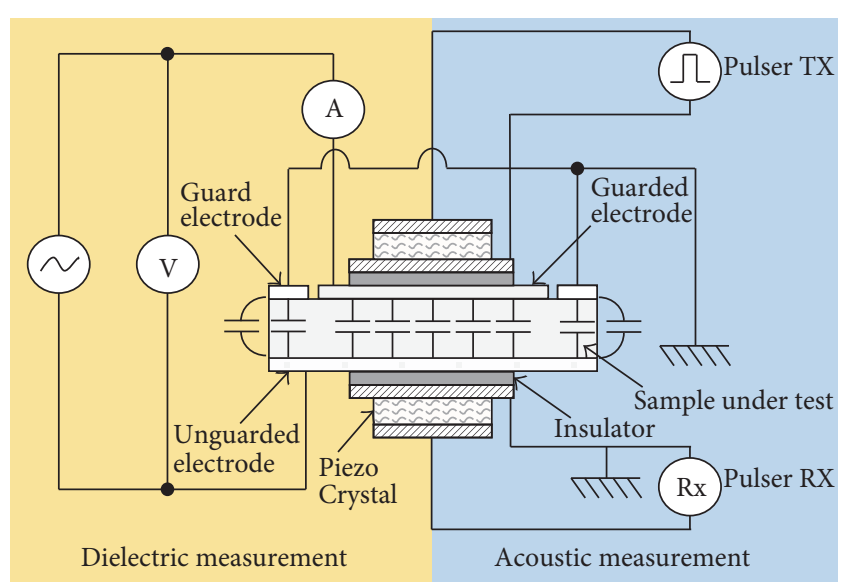

FIGURE 4: Principal scheme of the parallel plate measurement setup, modified from Josh [18].

average sample should not exceed one fifth of its diameter. The subsample discs were each placed in the parallel plate measurement cell where they were measured with an impedance analyser. The two following methods for running this device were employed. The bare coupling was used to measure the effective conductivity of the compacted samples. The disc subsample is simply placed between the parallel plate electrodes so they are in a direct contact with the bare faces of the subsample. For shales, this method provides a good conductive coupling between the rock surface and the electrodes. Coupling with insulating film was also used to block the current flow and enhance the relative contribution of polarization effects so that the frequency dispersion of real and imaginary parts of relative dielectric permittivity can be determined accurately.

Twelve artificial shale samples were prepared via the laboratory mechanical compaction. Dielectric analysis of the samples is conducted in two directions, normal (Figure 5(a)) and parallel to the bedding plane (Figure 5(b)), to investigate the dielectric anisotropy.

\section{Results and Discussion}

Dielectric properties are presented in Figures 6-13 for different parameters, namely, the salinity of pore fluid, its cation composition, clay content, and the type of initial clay microstructure. Figure 6 illustrates the positive linear trends of real dielectric permittivity versus porosity for the samples prepared with brine and fresh water separately. The dielectric response at frequencies of $>1 \mathrm{GHz}$ range is usually attributed to the dipole polarization of water molecules and might be used to estimate the amount of water in the pore space. Linear correlation decreases with a decrease in frequency from $R^{2}=$ 0.95 at $100 \mathrm{MHz}$ to $R^{2}=0.70$ at $10 \mathrm{MHz}$ due to contributions from other polarization mechanisms occurring at lower frequencies (e.g., surface-charge polarization). Although it is believed that the brine salinity has no effect on dielectric response of saturated rock samples at high frequencies, our experimental results show clearly separated trends for the two groups of the artificial shale samples prepared with brine and fresh water. This difference is attributable to the changes occurring in the electric double layer of clays in the presence of electrolyte. In the samples saturated with highly concentrated brine the positive ions of $\mathrm{Na}$ and $\mathrm{K}$ compensate the free charges on the surfaces of clay particles. Therefore, the diffuse layer of weakly bound water around the clay particles significantly shrinks $[20,21]$ and leaves more free water molecules that are easily polarizable within the sample. On the other hand, the samples prepared with fresh water exhibit thick hydrate envelopes around the clay particles thereby hindering the large amount of water dipoles from polarization in the presence of an electric field. In our experimental results the described effect results in one order of magnitude difference between the samples saturated with brine and fresh water samples on a wide range of porosity at megahertz frequencies. It is also important to note that the number of active cations in brine saturated samples seems to have negligible effect on dielectric permittivity in the dipole polarization frequency range as all the samples prepared with different concentrations and compositions of brine follow the same linear trends. Using the linear trends described above, it is possible to compare different samples at the same porosity. These trends are used to investigate the effects of clay content, salinity, and microstructure.

Figure 7 shows that across the wide range of frequencies horizontal conductivity in different samples is almost always higher than the vertical one. Previously we showed that the particles of clay and silt tend to orient with compaction normal to the direction of an applied compaction stress [16, 22]. Therefore, the interconnected pore network in artificial shale samples is also more oriented in the bedding direction and provides an effective pathway for charge carriers, whereas in the direction normal to bedding the conductive pathways exhibit greater tortuosity and obstruct the movement of charges. The conductivity anisotropy also increases with porosity reduction as the microstructure becomes more oriented in horizontal direction. Both horizontal and vertical conductivities increase with increasing porosity. At high frequencies, the influence of fluid conductivity grows with increase of the pore volume occupied with water solution.

The frequency dependent dielectric anisotropy in eight artificial shale samples is shown in Figure 8. Dashed line on the plot corresponds to the isotropic system, while all the points above and below this line correspond to anisotropic system with polarization effects dominating in horizontal and vertical directions, respectively. It is important to note that the fresh water samples are always better polarized in horizontal direction and reside above the isotropy line, whereas the brine saturated samples are better polarized in vertical direction at lower frequencies and exhibit inversion of the anisotropy with crossover points in megahertz range.

The anisotropy curves for all the samples exhibit peak values in megahertz range and their maximum values and frequency distributions are determined by the type and concentration of salt ions in the saturating fluid. Thus, the samples prepared with fresh water or low concentration fluids show the highest anisotropy peaks located at the lower end of megahertz range and then follow the three peaks at the 


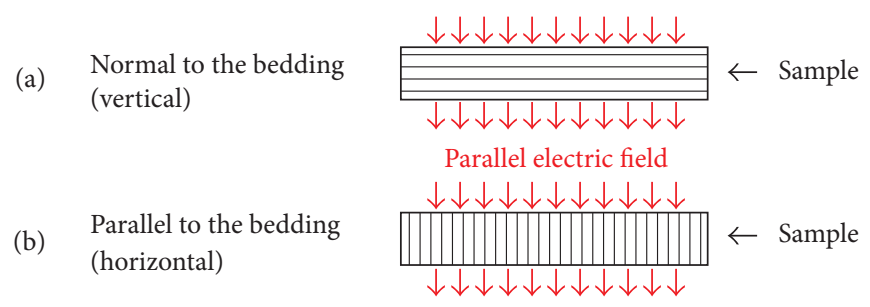

FIgURE 5: Parallel plate measurement schematics. Black lining illustrates the bedding of the sample.

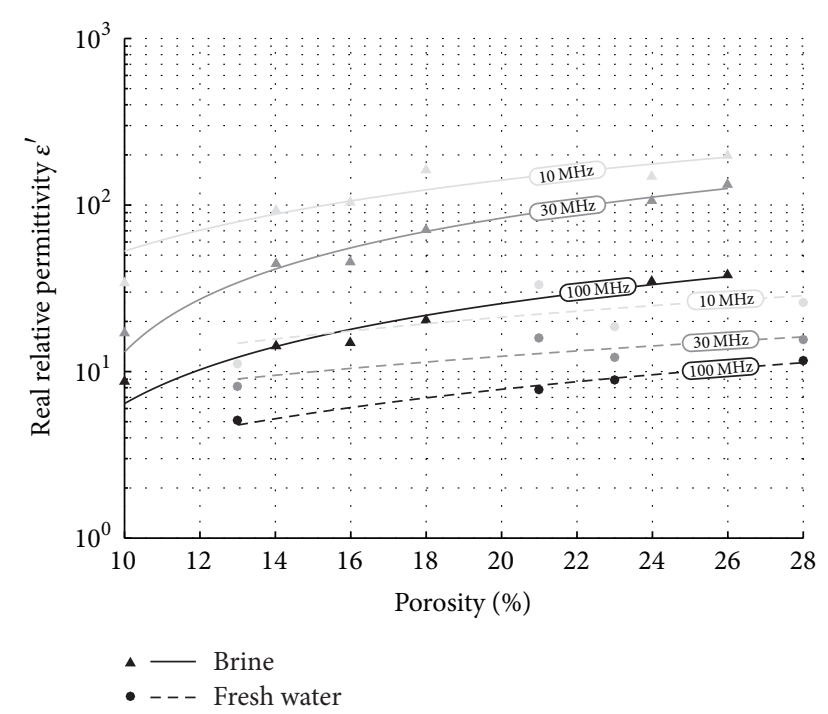

FIGURE 6: Dielectric response of brine saturated and fresh water samples at 100,30 , and $10 \mathrm{MHz}$.

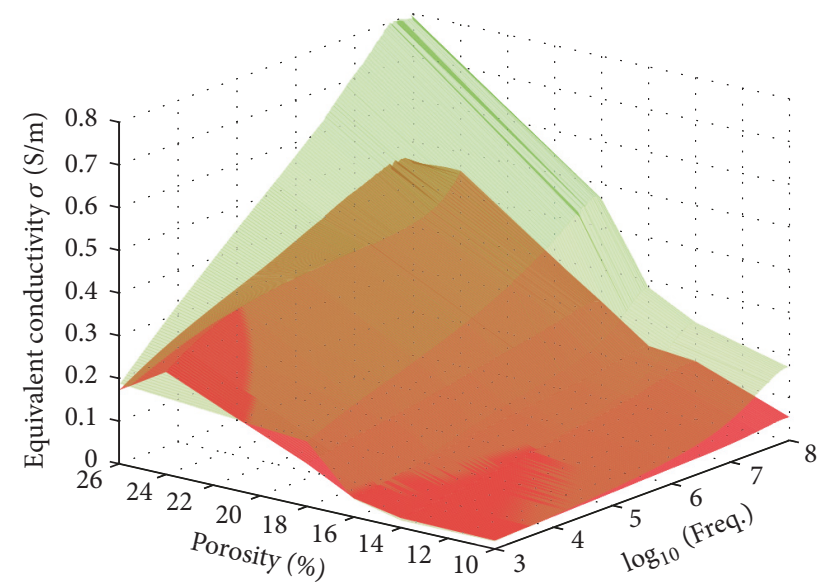

FigURE 7: Surface plot of conductivity, measured parallel (in green) and normal (in red) to the bedding, versus porosity and frequency in 6 brine saturated artificial shale samples.

same frequency of $\sim 60 \mathrm{MHz}$ corresponding to the samples saturated with $\mathrm{KCl}$ brine. The lowest peak belonging to the sample saturated with $\mathrm{NaCl}$ brine occurs at frequencies above $100 \mathrm{MHz}$. The crossover points with isotropy line in brine saturated samples are also distributed across the

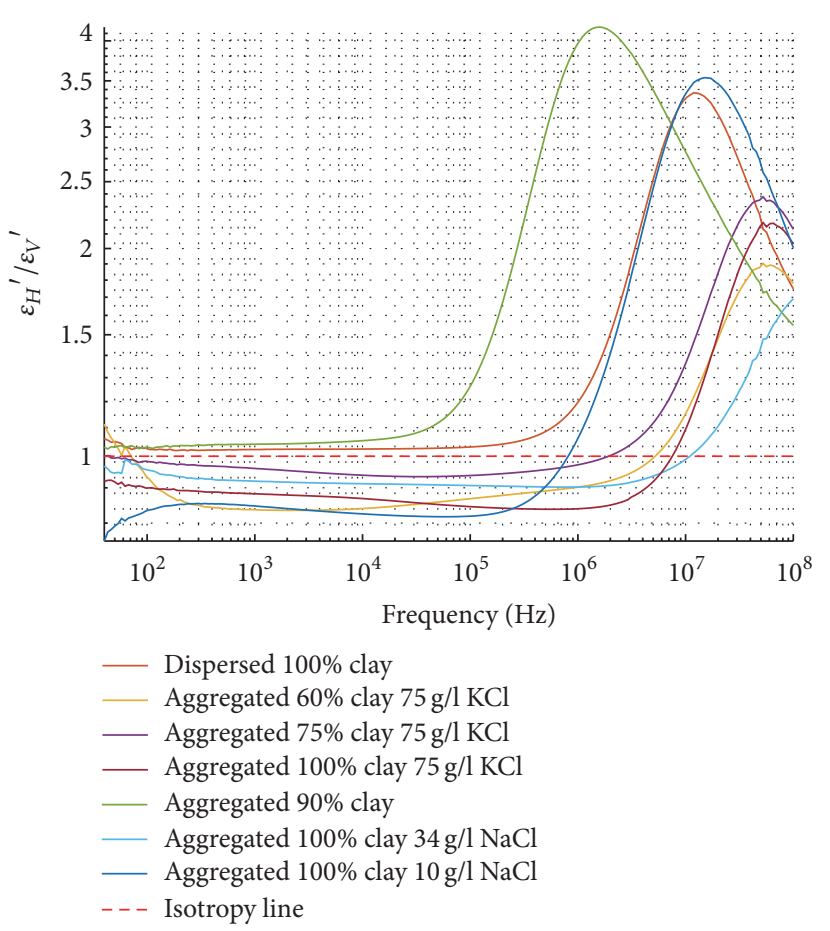

FIGURE 8: Dielectric anisotropy versus frequency in brine saturated and fresh water samples.

megahertz frequency, similarly to the peak values, but at lower frequencies.

This anisotropic behaviour may be explained with electric double layer theory. Given that the clay particles in fresh water samples have thick hydrate envelopes of weakly bound water, the dipole polarization of water molecules is achieved more easily in the parallel to bedding plane simply due to the fact that the clay particles in all the samples are mostly oriented in horizontal direction. Hence water molecules are oriented with their hydrogen atoms towards the surfaces of clay particles and are easily skewed sideways under the influence of electric field parallel to the bedding but resist the influence of normal electric field. In the brine saturated samples this effect is less pronounced across the wide frequency range due to the significantly thinner diffuse part of the double electric layers where the above phenomenon occurs. Therefore, considering the distinctive features of dielectric anisotropy in artificial samples, one might infer that the anisotropy analysis can help with understanding of composition and concentration of the saturating fluids in clay 


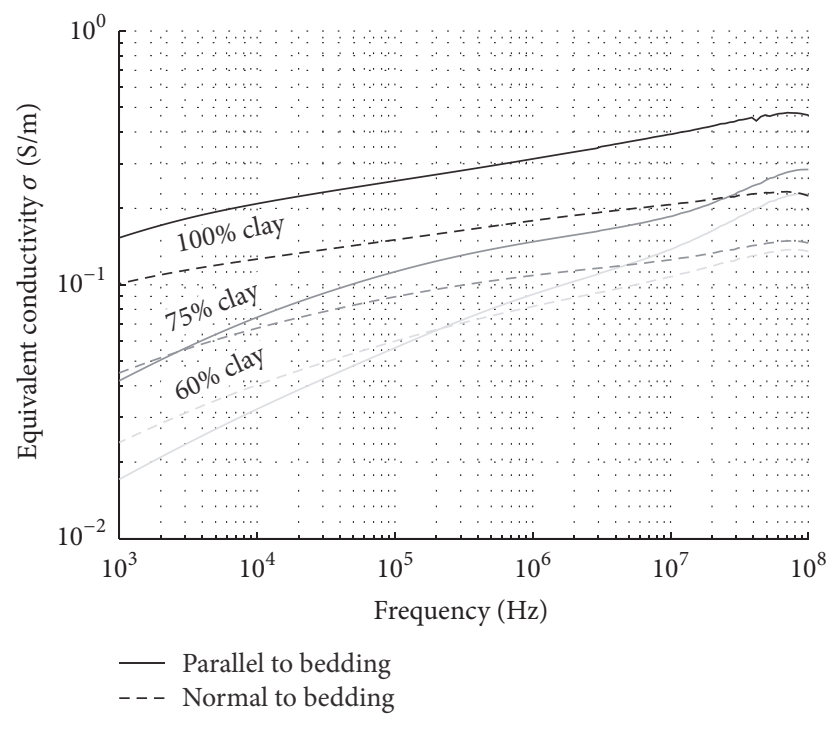

FIGURE 9: Effect of clay content on frequency dependent conductivity in samples measured parallel and normal to the bedding plane.

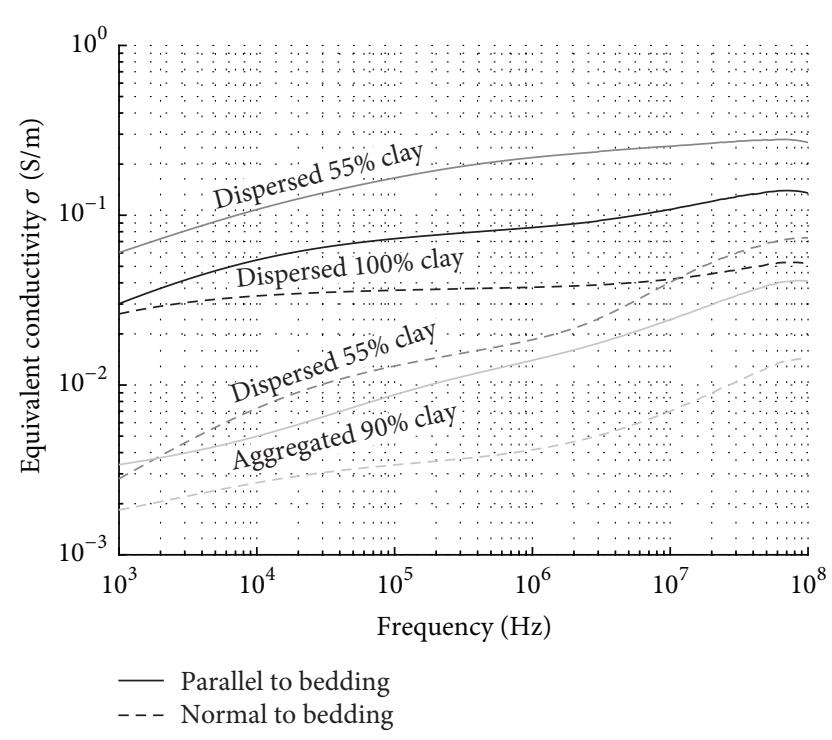

FIGURE 10: Effect of microstructure on conductivity of artificial shales measured parallel and normal to the bedding plane.

rocks. However, more data on artificial and natural rocks need to be analysed to confirm the discussed relationships.

Figure 9 shows that the increase of clay content at the same porosity results in greater conductivity in both vertical and horizontal directions. This behaviour is caused by the surface conduction mechanisms characteristic to clay particles. It has been shown that the counter ions located in the Stern layer of clay particles are the dominant contributors to surface conduction and in brine saturated rocks with salinity above $1 \mathrm{~mol} / \mathrm{l}$ the mobility of $\mathrm{K}$ and $\mathrm{Na}$ ions develops $1 / 10$ of that in the free fluid and is independent from the salinity [23]. Therefore, in our experiments replacing the fraction of quartz

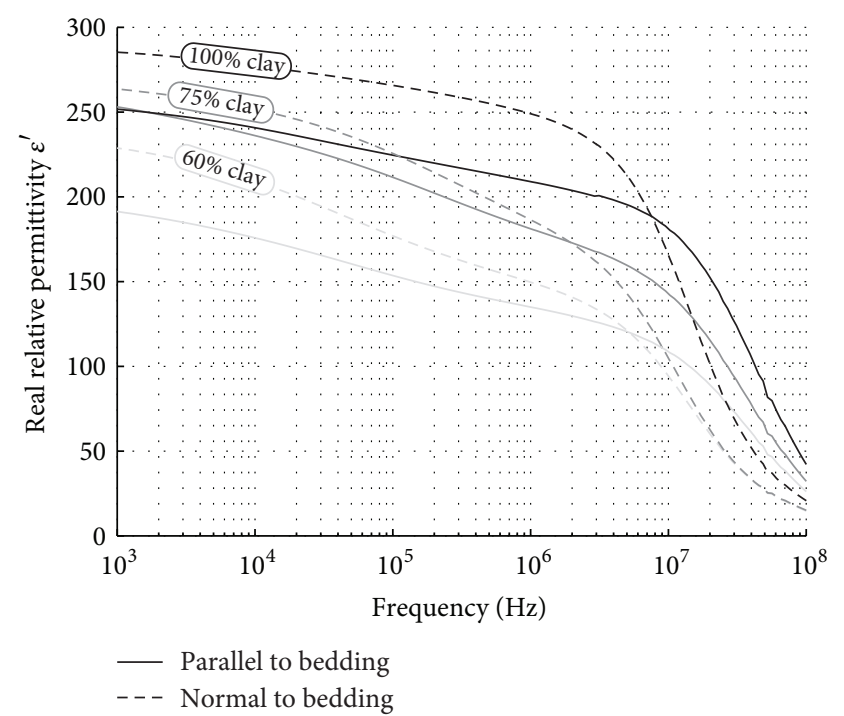

FIGURE 11: Dielectric response of the three brine-saturated artificial shale samples with clay contents of 60,75 , and 100 per cent.

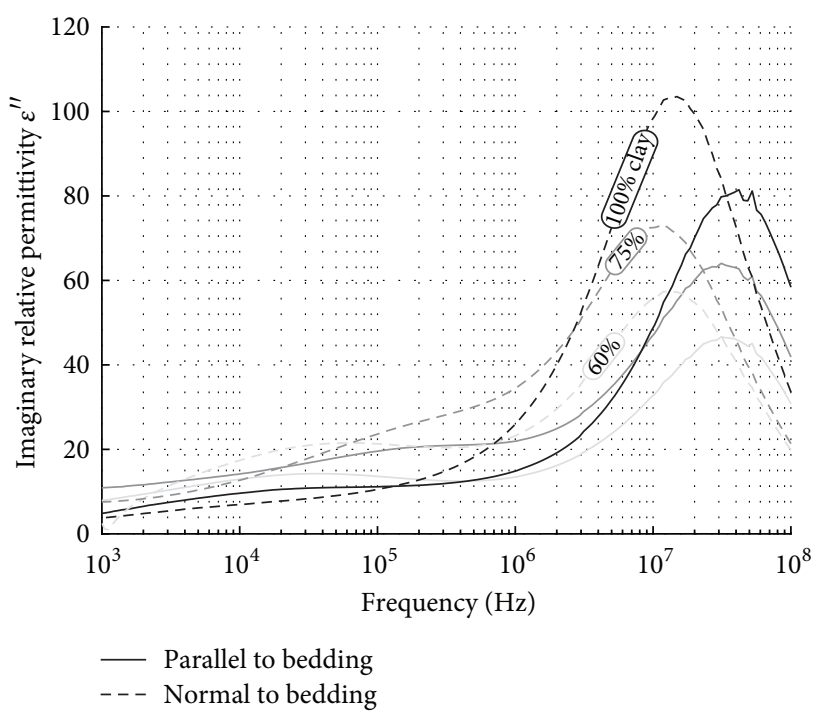

FIgURE 12: Frequency dependent dielectric loss in artificial shales with varying clay content.

with clay particles having much more uncompensated charge results in proportionally stronger surface conduction.

Figure 10 shows that the conductivity of the samples prepared with dispersed clay microstructure is always higher than that of the aggregated sample in both directions independently of the silt content and porosity. This is due to the greater surface conduction in the Stern layer of the dispersed samples where the clay particles are separated from each other and have greater free surface than their associations in aggregated sample.

At frequencies in the range of $1 \mathrm{kHz}$ to $100 \mathrm{MHz}$ the vertical polarization dominates in the brine saturated samples as shown in Figure 11. The main polarization mechanism in 


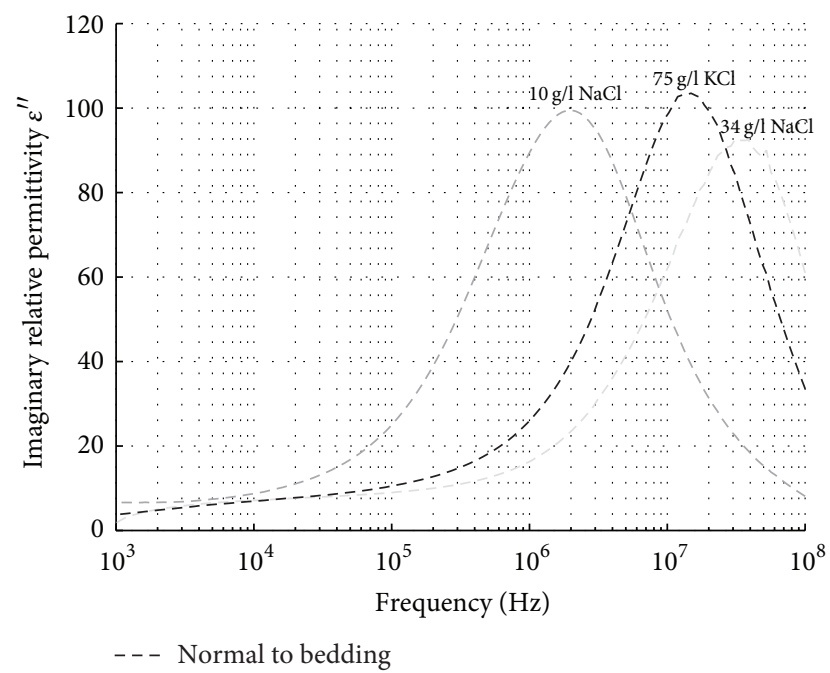

FIgURE 13: Frequency dependent dielectric loss in brine saturated artificial shales.

this frequency band is the Maxwell-Wagner polarization of counter ions. The movement of ions in the vertical direction is restricted due to the strong orientation of clay particles normal to the applied electric field and the force balance within their electric double layers. In this case each individual clay particle acts as a capacitor and the ensemble of such particles gives rise to strong polarization effects exceeding that of the individual fluid and mineral phases [3]. In contrast, the cations can be easily drawn along the surface of clay platelets and through the less tortuous pore network along the bedding. Thus, in the presence of a low frequency electric field (below megahertz) in the direction normal to the bedding hydrated counter ions are prone to polarization rather than to conduction and vice versa for the electric field in the bedding direction. Also, it is important to note that the higher the clay content is the more the polarization effects are pronounced in both directions due to the higher concentration and the better alignment of clay platelets given the same porosity level [16].

Figure 11 shows the rollover of real relative permittivity in the $\mathrm{MHz}$ range of frequencies for both the vertical and horizontal measurements. According to Kramers-Krönig relationship [24] these rollovers correspond to the peaks on the plots of imaginary relative permittivity in Figure 12. Peak values of dielectric loss-factor in vertical direction appear at approximately one order of magnitude lower frequencies and are $\sim 20 \%$ higher than those measured in horizontal direction. One must take these effects into account designing the borehole heating antennas for effective hydrocarbon extraction in unconventional reservoirs. Also, samples with higher clay content show the higher values of loss-factor due to the sharper rollover in the real relative permittivity caused by the change in polarization mechanism from surfacecharge to dipole polarization with increase of frequency.

Figure 13 illustrates the dielectric loss-factor for the three samples saturated with different brine cation composition and concentration. All the peaks are located at $\mathrm{MHz}$ range for the measured samples. Distribution of the peaks at different frequencies depends on the salinity of a pore fluid and its cation composition. Hence, increasing the salinity from 10 to $34 \mathrm{~g} / \mathrm{l}$ in pure kaolinite sample saturated with $\mathrm{NaCl}$ results in change of peak frequency from $2 \times 10^{6}$ to $3 \times 10^{7} \mathrm{~Hz}$. Sample saturated with $75 \mathrm{~g} / \mathrm{l} \mathrm{NaCl}$ solution exhibits the peak frequency of $4 \times 10^{7}$ which highlights the effect of different salt ions on the dielectric loss-factor of clay rocks.

\section{Conclusions}

Artificial shales with simple mineral composition illustrate the broad frequency dispersion of dielectric effects. The variations in salinity of the connate water, its cation composition, clay content, and microstructure of artificial shales significantly affect complex dielectric permittivity and conductivity of artificial shales. These effects can be explained with the Maxwell-Wagner polarization effects at frequencies below megahertz range and the changes occurring in double electric layer of clay particles in the presence of electrolyte at higher frequencies.

It is shown that at high frequencies (above $10 \mathrm{MHz}$ ) real relative permittivity has different linear trends with porosity in fresh water and brine saturated samples. The salinity and cation composition of the pore fluid seem to have negligible effect on these high frequency dielectric trends.

Formation of anisotropic microstructure of artificial shales during mechanical compaction results in significant values of dielectric anisotropy between 2 and 4 . The magnitude and characteristic frequency of the peak values in anisotropy curves as well as the crossover with isotropy point is dependent on salinity, cation composition of saturating fluid, and the clay content of the samples.

The absolute peak value of dielectric loss in shales and its characteristic frequency depends not only on the amount of connate fluid, but also on the cation composition of the saturating brine, its salinity, and the orientation of an applied electric field relative to the shale bedding. The peak values of the dielectric loss measured along and normal to bedding lay within the megahertz frequency range with significant separation of approximately 1 order of magnitude. The absolute values of these peaks are approximately 20 percent higher in the direction normal to the bedding.

Our simplistic models of naturals shales illustrated the complex dielectric behaviour similar to that of the real rocks. The theoretical modelling of dielectric response is conducted in companion paper illustrating the use of artificial shales for design and calibration of the rock physics models.

\section{Conflicts of Interest}

The authors declare that they have no conflicts of interest.

\section{Acknowledgments}

The authors would like to thank the CSIRO Shale Rock Physics and Petrophysics (SHARPP) consortium sponsored by Total for financial support of this work. R. Beloborodov would like to acknowledge ASEG Research Foundation for sponsoring him with Grant RF16P05. 


\section{References}

[1] A. R. von Hippel, Dielectrics and Waves, Wiley, New York, NY, USA; Chapman \& Hall, London, UK, 1954.

[2] P. N. Sen, "Relation of certain geometrical features to the dielectric anomaly of rocks," Geophysics, vol. 46, no. 12, pp.1714$1720,1981$.

[3] P. N. Sen and W. C. Chew, "The frequency dependent dielectric and conductivity response of sedimentary rocks," The Journal of Microwave Power and Electromagnetic Energy, vol. 18, no. 1, pp. 95-105, 1983.

[4] D. Freed, N. Seleznev, C.-Y. Hou et al., "A physics-based model for the dielectric response of shaly sands," in Proceedings of the 57th Annual Logging Symposium (SPWLA '16), Society of Petrophysicists and Well-Log Analysts, Reykjavik, Iceland, June 2016.

[5] M. Hizem, H. Budan, B. Devillé, O. Faivre, L. Mossé, and M. Simon, "Dielectric dispersion: a new wireline petrophysical measurement," in Proceedings of the SPE Annual Technical Conference and Exhibition (ATCE '08), pp. 3230-3250, Society of Petroleum Engineers, Denver, Colo, USA, September 2008.

[6] M. Josh, M. B. Clennell, M. D. Raven, and D. N. Dewhurst, "Factors controlling dielectric response of clays and shales," in Proceedings of the 2015 SEG Annual Meeting, Society of Exploration Geophysicists, New Orleans, Louisiana, October 2015.

[7] A. A. Garrouch and M. M. Sharma, "Influence of clay content, salinity, stress, and wettability on the dielectric properties of brine-saturated rocks: $10 \mathrm{~Hz}$ to $10 \mathrm{MHz}$," Geophysics, vol. 59, no. 6, pp. 909-917, 1994.

[8] P. N. Sen, "The dielectric and conductivity response of sedimentary rocks," in Proceedings of the SPE Annual Technical Conference and Exhibition, Society of Petroleum Engineers, Dallas, Tex, USA, September 1980.

[9] A. Mukhametshina and E. Martynova, "Electromagnetic heating of heavy oil and bitumen: a review of experimental studies and field applications," Journal of Petroleum Engineering, vol. 2013, Article ID 476519, 7 pages, 2013.

[10] T. Abraham, C. W. Van Neste, A. Afacan, and T. Thundat, "Dielectric relaxation-based capacitive heating of oil sands," ENERGY \& FUELS, vol. 30, no. 3, pp. 1987-1996, 2016.

[11] M. Josh, "Dielectric permittivity: a petrophysical parameter for shales," Petrophysics, vol. 55, pp. 319-332, 2014.

[12] J. G. Berryman and G. M. Hoversten, "Modelling electrical conductivity for earth media with macroscopic fluid-filled fractures," Geophysical Prospecting, vol. 61, no. 2, pp. 471-493, 2013.

[13] M. Al-Harahsheh, S. Kingman, A. Saeid, J. Robinson, G. Dimitrakis, and H. Alnawafleh, "Dielectric properties of Jordanian oil shales," Fuel Processing Technology, vol. 90, no. 10, pp. 12591264, 2009.

[14] J.-M. Donadille, "On the petrophysical analysis of dielectric anisotropy," in Proceedings of the 57th Annual Logging Symposium (SPWLA '16), Society of Petrophysicists and Well-Log Analysts, Reykjavik, Iceland, June 2016.

[15] M. Josh, B. Clennell, and M. Cauchefert, "Dielectric permittivity and anisotropy of intact multi-saturated organic shales," in Proceedings of the 57th Annual Logging Symposium (SPWLA '16), Society of Petrophysicists and Well-Log Analysts, Reykjavik, Iceland, June 2016.

[16] R. Beloborodov, M. Pervukhina, V. Luzin et al., "Compaction of quartz-kaolinite mixtures: the influence of the pore fluid composition on the development of their microstructure and elastic anisotropy," Marine and Petroleum Geology, vol. 78, pp. 426-438, 2016.

[17] X. Dong and Y.-H. Wang, “The effects of the $\mathrm{pH}$-influenced structure on the dielectric properties of kaolinite-water mixtures," Soil Science Society of America Journal, vol. 72, no. 6, pp. 1532-1541, 2008.

[18] M. Josh, "A combination dielectric and acoustic laboratory instrument for petrophysics," Measurement Science and Technology, vol. 28, no. 12, Article ID 125904, 2017.

[19] V. I. Osipov, V. N. Sokolov, and V. V. Eremeev, Clay Seals of Oil and Gas Deposits, A. A. Balkema Publishers, Lisse, the Netherlands, 2004.

[20] K. Bohinc, V. Kralj-Iglič, and A. Iglič, "Thickness of electrical double layer. Effect of ion size," Electrochimica Acta, vol. 46, no. 19, pp. 3033-3040, 2001.

[21] M. A. Brown, A. Goel, and Z. Abbas, "Effect of electrolyte concentration on the stern layer thickness at a charged interface," Angewandte Chemie International Edition, vol. 55, no. 11, pp. 3790-3794, 2016.

[22] R. Beloborodov, M. Pervukhina, L. Esteban, and M. Lebedev, "Compaction of quartz-kaolinite powders with aggregated initial microstructure-elastic properties and anisotropy," in Proceedings of the 77th EAGE Conference and Exhibition 2015-Earth Science for Energy and Environment, Madrid, Spain, June 2015.

[23] A. Revil and P. W. J. Glover, "Nature of surface electrical conductivity in natural sands, sandstones, and clays," Geophysical Research Letters, vol. 25, no. 5, pp. 691-694, 1998.

[24] F. Kremer and A. Schönhals, Broadband Dielectric Spectroscopy, Springer, Berlin, Germany, 2002. 

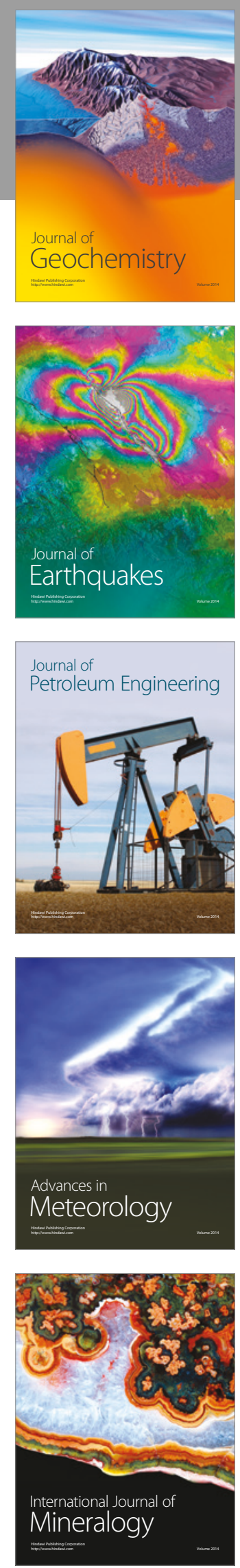
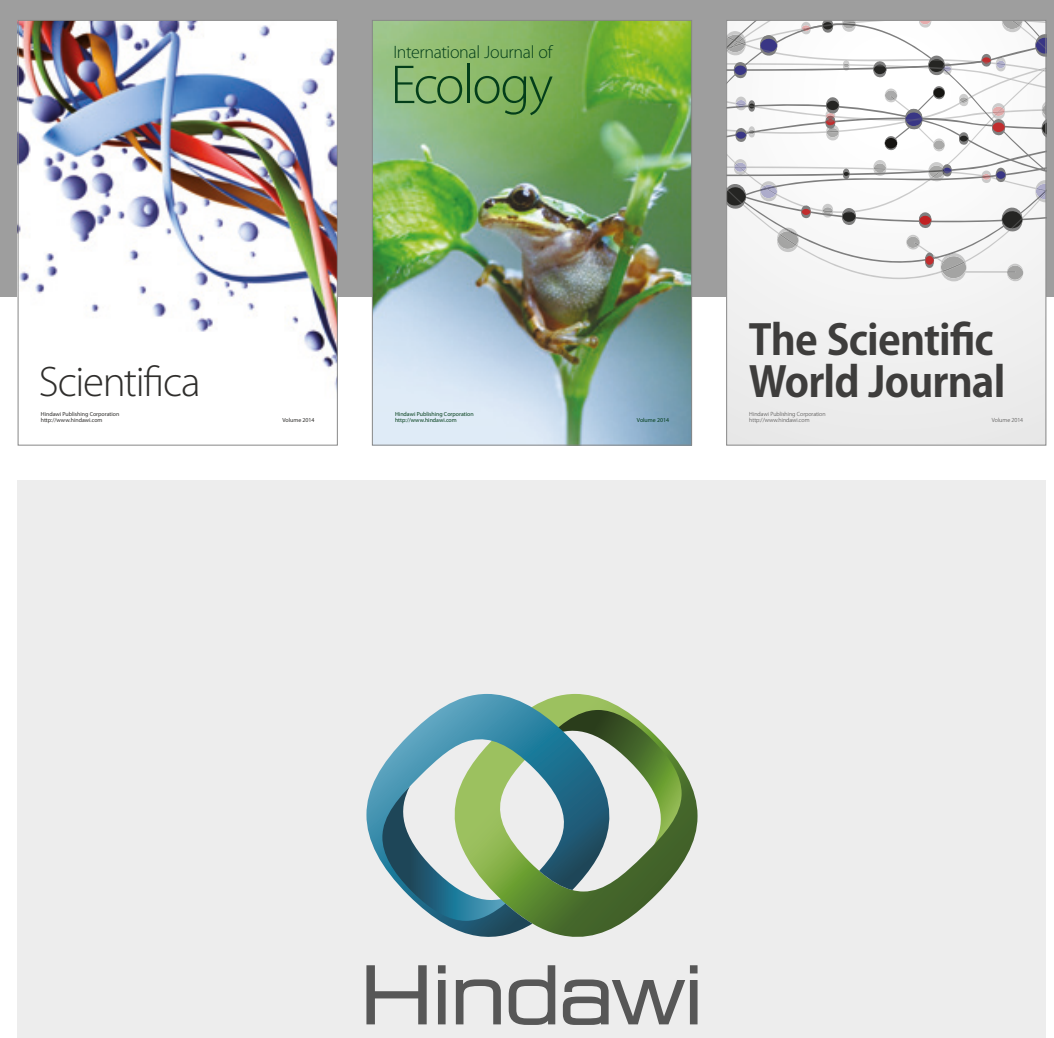

Submit your manuscripts at

https://www.hindawi.com
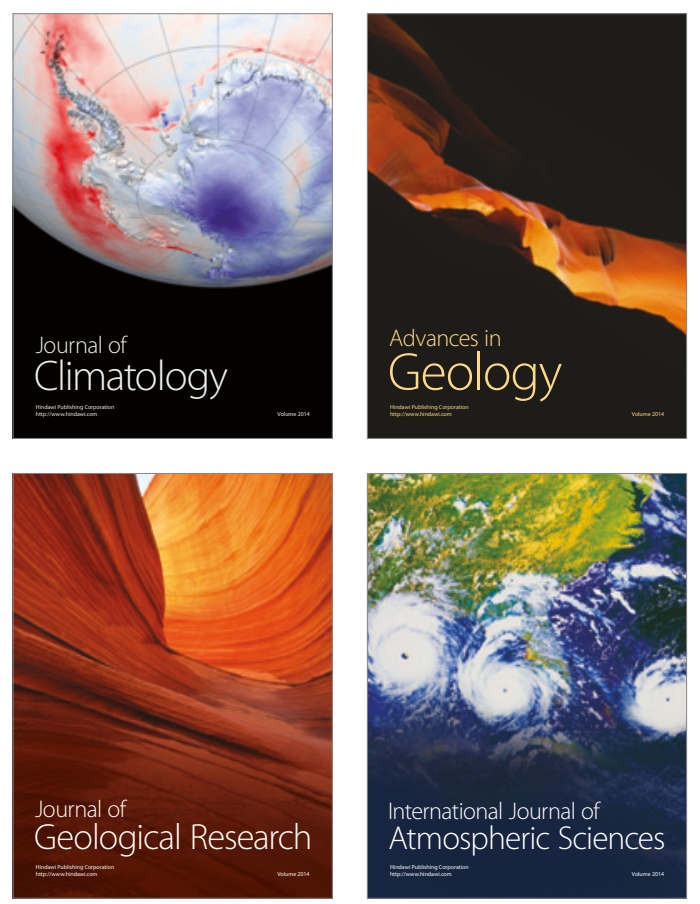

The Scientific

World Journal
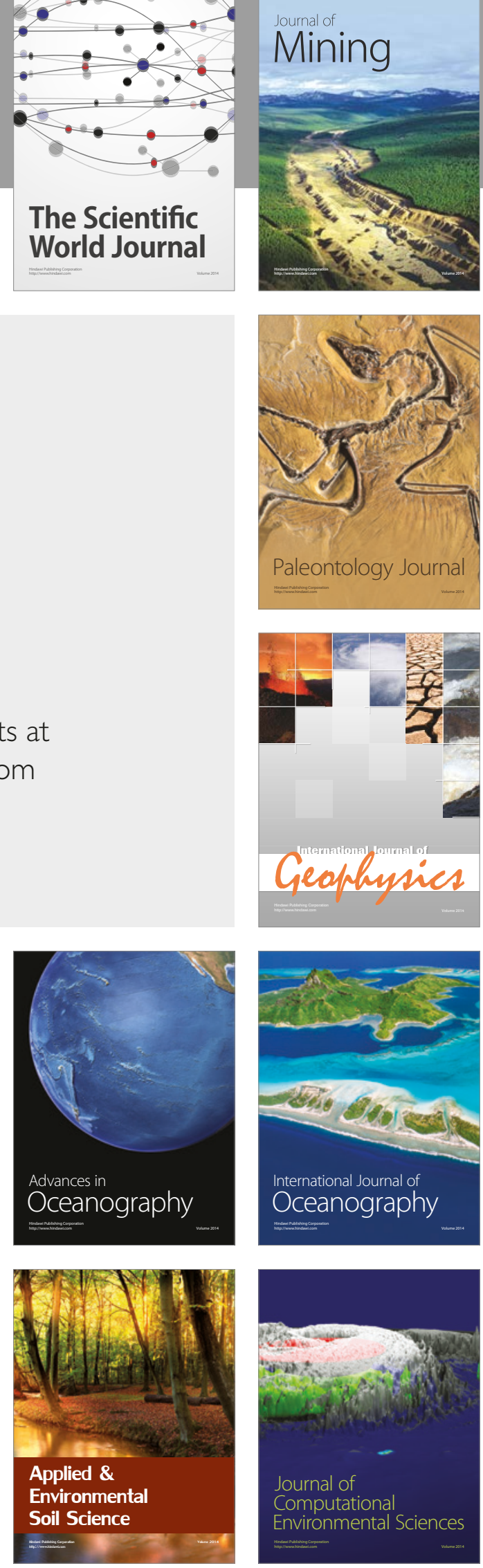